\title{
ALTERAÇÃO EXPERIMENTAL DE ROCHAS CARBONÁTICAS, CAVERNA DAS PERÓLAS, IPORANGA (SP)
}

\author{
Mirian Chieko SHINZATO \\ Raphael HYPOLITO \\ Alex José BARBIERI \\ Flavio Machado de Souza CARVALHO
}

\begin{abstract}
RESUMO
Trabalhos experimentais foram realizados com o intuito de estudar a alteração química em rochas carbonáticas provenientes da Caverna das Pérolas, Município de Iporanga, Estado de São Paulo. Extratores soxhlet foram utilizados para acelerar o processo de lixiviação de duas formas: contínua e intermitente. A solução de lixiviação obtida no ensaio contínuo foi analisada quimicamente no final do período de lixiviação (cerca de 454 horas), enquanto os precipitados foram separados e analisados mineralogicamente por difração de raios X (DRX). No ensaio de alteração intermitente, as soluções foram recolhidas após períodos pré-determinados e analisados quimicamente junto com os precipitados, para determinar o total de íons remobilizados da rocha alterada. A composição química das rochas alteradas experimentalmente também foi analisada e comparada com a da rocha sã, a partir das frações molares de seus constituintes maiores. Verificou-se que os principais íons lixiviados no início do processo de alteração foram $\mathrm{Na}^{+} \mathrm{e} \mathrm{Mg}^{2+}$; no entanto, com o aumento da alteração química, os íons $\mathrm{Sr}^{2+}, \mathrm{Ba}^{2+} \mathrm{e} \mathrm{Ca}^{2+}$ passaram a ser, juntamente com o $\mathrm{Na}^{+}$, os íons mais remobilizados, dando início também à precipitação de calcita. Observou-se que no experimento contínuo as concentrações de $\mathrm{Sr}^{2+}$ e $\mathrm{Ba}^{2+}$ ficaram inferiores em relação ao experimento intermitente, revelando que esses íons possivelmente co-precipitaram com a calcita. A baixa razão $\mathrm{Mg} / \mathrm{Ca}$ pode ter promovido a precipitação preferencial da calcita, em relação à aragonita, nas soluções de alteração experimental. Verificou-se que houve maior taxa de remobilização dos constituintes da rocha carbonática na zona em que as amostras foram submetidas a flutuações do nível freático no compartimento do soxhlet. Entre os minerais secundários observados na rocha alterada, destaca-se a caulinita.
\end{abstract}

Palavras-chave: alteração experimental, soxhlet, lixiviação, rochas carbonáticas.

\section{ABSTRACT}

Experimental studies were performed to study the chemical weathering of carbonate rocks from the Pérolas Cave, Iporanga municipality, State of São Paulo. Soxhlet extractors were used to accelerate the leaching process in two different ways: continuous and intermittent. The leaching solution obtained from the continuous test was chemically analyzed at the end of the process (about 454 hours), and the precipitates were separated and mineralogically analyzed by X-ray diffraction (XRD). In the intermittent leaching process, solutions and precipitates were collected after a predetermined period of time and chemically analysed to determine the number of ions removed from the weathered rocks. It was found that the ions mainly leached in the beginning of the experimental weathering were $\mathrm{Na}^{+}$and $\mathrm{Mg}^{2+}$; however, when the leaching process was accelerated, $\mathrm{Sr}^{2+}, \mathrm{Ba}^{2+}$ and $\mathrm{Ca}^{2+}$ became the most remobilized ions, along with $\mathrm{Na}^{+}$, leading to the precipitation of calcite. In the continuous leaching test, it was observed that the concentrations of $\mathrm{Sr}^{2+}$ and $\mathrm{Ba}^{2+}$ were lower compared to the intermittent test, showing that these ions were probably co-precipitated with calcite. The low $\mathrm{Mg} / \mathrm{Ca}$ ratio in the experimental leaching solutions may have caused the precipitation of calcite instead of aragonite. It was noted that there was a higher 
rate of remobilization of the constituents of the carbonate rocks on the level where the samples were subjected to fluctuations of the water table in the compartment of the Soxhlet equipment. Kaolinite is the main secondary mineral in the weathered rock.

Keywords: experimental weathering, soxhlet, leaching process, carbonate rocks.

\section{INTRODUÇÃO}

Trabalhos experimentais de lixiviação ou alteração intempérica são importantes por permitirem acelerar e controlar alguns parâmetros envolvidos no processo de intemperismo de rochas. Os resultados desses estudos podem indicar, por exemplo, os tipos de reação ou decomposição química dos minerais, os principais íons remobilizados e, ainda, as transformações mineralógicas ocorridas durante o processo.

Os métodos utilizados nesses estudos consistem basicamente na inserção de fragmentos de rochas em recipientes ou colunas preenchidas e/ou percoladas com água, ou outra solução de interesse, que é posteriormente analisada. Para simular a chuva ácida, por exemplo, pode-se utilizar soluções ácidas (SIMÃO \& SILVA 1999, KRYZA et al. 2009), ou mesmo, água destilada saturada com $\mathrm{CO}_{2}$ (MARTINEZ \& WHITE 1999, HOREAU et al. 2005).

Diferentes tipos de instrumentos ou equipamentos também têm sido empregados nesse tipo de trabalho, como o extrator soxhlet utilizado por PEDRO (1964) em suas investigações sobre alteração de rochas. Desde então novas abordagens vem sendo exploradas nessa área (HYPOLITO 1974), uma vez que esses equipamentos permitem controlar o tempo e o número de ciclos de lixiviação. Nessa linha, vários materiais geológicos têm sido investigados, como rochas vulcânicas (CUADROS et al. 1999, GOMES 2001), granulitos (COSTA et al. 2010), anortositos (SIMÃO \& SILVA 1999) e até mesmo meteoritos condríticos ordinários (LEE et al. 2006).

Cabe ressaltar que, pelo fato do extrator soxhlet utilizar soluções de percolação a altas temperaturas (entre $60-80{ }^{\circ} \mathrm{C}$ ), as condições experimentais não representam àquelas observadas na natureza. No entanto, por promover de forma acelerada a alteração de uma rocha em meio aquoso, os resultados podem revelar a predisposição de alguns minerais à dissolução durante o intemperismo químico. Os dados analíticos das soluções de lixiviação também podem fornecer a ordem de mobilidade relativa dos íons solubilizados em um determinado período de tempo. Dependendo da concentração e da natureza dos íons lixiviados, pode-se ainda promover condições para a precipitação de novos minerais.
Existem vários estudos sobre alteração experimental de rochas silicáticas, como os de HYPOLITO (1974), KOJIMA et al. (1997), WHITE et al. (1999), WU et al. (2001), WHITE \& BRANTLEY (2003), PRIKRYL et al. (2003) e FRASCÁ \& YAMAMOTO (2006); no entanto, são poucos os que procuram estudar, em laboratório a alteração de rochas carbonáticas. Vários parâmetros interferem na dissolução dessas rochas, como pH da solução de percolação, composição química, tempo, temperatura etc., assim como em sua precipitação (efeito da força iônica da solução, pressão de $\mathrm{CO}_{2}$ etc.) (FORD \& WILLIAMS 2007).

De acordo com DREYBRODT et al. (1996) a dissolução do $\mathrm{CaCO}_{3}$ num sistema formado por $\mathrm{H}_{2} \mathrm{O}-\mathrm{CO}_{2}-\mathrm{CaCO}_{3}$ é controlado por três processos: (1) cinética de dissolução da superfície do mineral, (2) transporte de massa por difusão e, (3) cinética de reação do sistema $\mathrm{H}_{2} \mathrm{O}+\mathrm{CO}_{2}=\mathrm{H}^{+}+\mathrm{HCO}_{3}^{-}$.

O modelo teórico de BUHMANN \& DREYBRODT (1985) prediz ainda que a razão de dissolução depende da razão $V / A$ (onde $V$ é o volume da solução e $A$, a área superficial do mineral). De acordo com os autores, se o volume da solução percolante aumentar, a ponto da quantidade de $\mathrm{CO}_{2}$ consumido durante a dissolução for insuficiente para mudar sua concentração, então a superfície de reação ou o transporte de massa determinarão a razão de dissolução. Dessa forma, a cinética de conversão de $\mathrm{CO}_{2}$ torna-se importante em todos os processos geológicos, onde grandes áreas da superfície mineral estão em contato com pequenos volumes de água (DREYBRODT et al. 1996).

Assim sendo, dois sistemas podem ser definidos para as condições de dissolução ou precipitação da calcita. No sistema aberto a solução deve estar em contato com a atmosfera, que fornecerá um fluxo contínuo de $\mathrm{CO}_{2}$ para a solução. Caso haja somente consumo de $\mathrm{CO}_{2}$, o sistema será fechado (DREYBRODT \& BUHMANN 1991).

Nesse trabalho, testes de alteração acelerada utilizando o extrator soxhlet foram realizados em rochas carbonáticas da Caverna das Pérolas, Município de Iporanga (SP). Este estudo teve a finalidade de examinar as características químicas e mineralógicas das rochas alteradas e dos minerais precipitados na solução de lixiviação, bem como inferir o comportamento geoquímico dos elementos remobilizados durante o processo de intemperismo num sistema aberto. 


\section{MATERIAL E MÉTODOS}

As rochas carbonáticas utilizadas nesse estudo foram coletadas na Caverna das Pérolas, $\mathrm{Mu}$ nicípio de Iporanga (SP). Essa caverna está inserida no contexto da Província Espeleológica do Alto Ribeira (KARMANN \& SANCHEZ 1979). Geologicamente, tais rochas pertencem à Formação Bairro da Serra do Subgrupo Lajeado, Grupo Açungui (CAMPANHA 1991) que é composta por mármores finos homogêneos, por vezes bandados (bandas decimétricas a centimétricas). Em geral são calcíticos e, localmente, dolomíticos, impuros, margosos, passando a filito e xistos carbonáticos, com intercalações de filitos sericíticos e metassil- titos. Apresentam também pontuações de sulfetos (pirita) disseminados na rocha (IPT 1984).

Segundo BARBIERI (1993) a Caverna das Pérolas desenvolve-se principalmente ao longo do atual nível freático, definido pelo Rio Roncador, e apresenta vários salões representando paleocondutos freáticos. Alguns salões encontram-se extremamente ornamentados por estalactites, estalagmites, helictites, destacando-se ainda, a presença de concreções com formas elipsoidais e esféricas, conhecidas como "pérolas".

As rochas carbonáticas coletadas na Caverna das Pérolas são constituídas principalmente por calcita, dolomita, quartzo e muscovita (BARBIERI 1993); sua composição química encontra-se descrita na Tabela 1.

Tabela1 - Principais óxidos e elementos-traços que compõe a rocha carbonática da Caverna das Pérolas (Fonte: BARBIERI, 1993).

\begin{tabular}{|c|c|c|c|c|c|c|c|c|c|c|}
\hline \multicolumn{11}{|c|}{ Principais óxidos (\%) } \\
\hline $\mathrm{CaO}$ & $\mathrm{MgO}$ & $\mathrm{SiO}_{2}$ & $\mathrm{Fe}_{2} \mathrm{O}_{3}$ & $\mathrm{TiO}_{2}$ & $\mathrm{BaO}$ & $\mathrm{K}_{2} \mathrm{O}$ & $\mathrm{Na}_{2} \mathrm{O}$ & $\mathrm{Al}_{2} \mathrm{O}_{3}$ & $\mathrm{SrO}$ & $P F$ \\
\hline 48,88 & 2,79 & 4,64 & $\mathrm{Nd}$ & 0,05 & 0,14 & 0,24 & 0,10 & 1,62 & 0,19 & 40,59 \\
\hline \multicolumn{11}{|c|}{ Elementos-traços $\left(\mathrm{mg} \cdot \mathrm{kg}^{-1}\right)$} \\
\hline \multicolumn{2}{|c|}{$C u$} & \multicolumn{2}{|c|}{$P b$} & \multicolumn{2}{|l|}{$M n$} & \multicolumn{2}{|l|}{ Co } & \multicolumn{3}{|l|}{$\mathrm{Ni}$} \\
\hline \multicolumn{2}{|c|}{12} & \multicolumn{2}{|c|}{56} & \multicolumn{2}{|l|}{125} & \multicolumn{2}{|l|}{20} & \multicolumn{2}{|l|}{40} & 367 \\
\hline
\end{tabular}

No estudo de alteração experimental utilizou-se o extrator do tipo soxhlet, constituído, da base para o topo, por uma manta aquecedora, balão de vidro com solução de lixiviação, tubo extrator e tubo condensador (Figura 1).

A lixiviação ocorre quando a solução contida no balão é aquecida pela manta produzindo vapor, que é conduzido ao topo do tubo extrator, onde é condensado. Assim, as gotas produzidas pela condensação percolam as rochas no interior de um cartucho poroso e, gradativamente, o tubo extrator é preenchido pela solução até alcançar o nível de um sifão, que conduz a solução de lixiviação de volta para o balão, reiniciando todo o processo.

Antes de serem introduzidas no soxhlet, as rochas foram trituradas e selecionadas em peneiras na granulometria entre 2 e $6 \mathrm{~mm}$, lavadas com água destilada e secas em estufa à $60^{\circ} \mathrm{C}$ por $24 \mathrm{~h}$. Em seguida, o cartucho com as amostras foi colocado no fundo do tubo extrator do soxhlet, de modo que a parte superior do cartucho (A) permanecesse acima do nível d'água, a parte intermediária (B) ficasse submetida à variação desse nível e a porção inferior (C), submersa a maior parte do tempo.

$\mathrm{Na}$ extremidade superior do aparato, próximo do local do gotejamento da água, foi introduzido

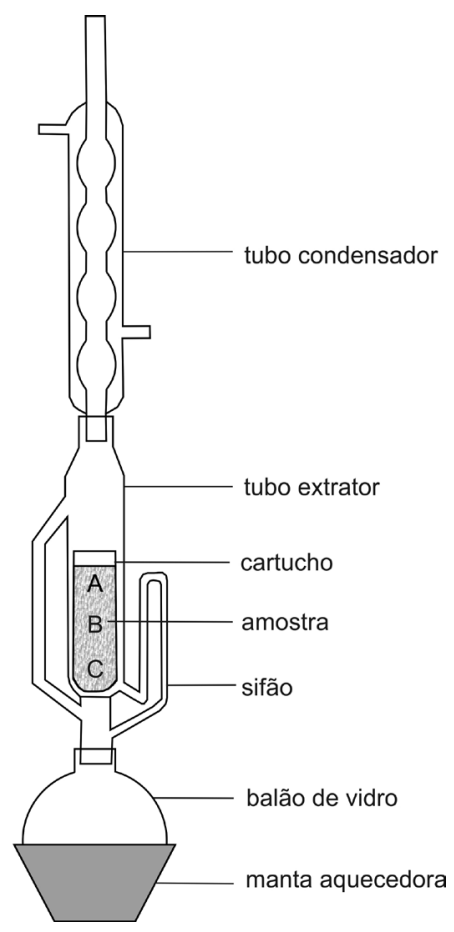

Figura 1 - Figura esquemática do extrator soxhlet. A: zona aerada, B: zona intermediária (sujeita a flutuações do nível de água) e C: zona submersa. 
um dispositivo para injetar gás carbônico, com o intuito de simular uma chuva ácida e, consequentemente, acelerar o processo de alteração química.

Os ensaios de alteração (AM-1 e AM-2) foram realizados sob condições distintas, descritas a seguir.

\subsection{AM-1 - Ensaio de alteração contínua}

Nesse ensaio, o soxhlet permaneceu funcionando continuamente durante 454 horas, resultando em uma única solução, que foi analisada quimicamente por absorção atômica (ASS-3 da Carl Zeiss) e por fotometria de chama (modelo B295II da Micronal). Foram inseridos dentro do cartucho $300 \mathrm{~g}$ de rocha e $850 \mathrm{~mL}$ de água destilada no balão de fundo redondo. Cada etapa de lixiviação levou cerca de 2 horas para encher o compartimento superior, onde se encontrava o cartucho com a amostra; o volume da solução de percolação envolvido em cada ciclo de lixiviação foi em torno de $250 \mathrm{~mL}$. A temperatura aproximada no interior do soxhlet foi de $85{ }^{\circ} \mathrm{C}$. A solução final foi filtrada e os materiais precipitados no fundo do balão de vidro foram recolhidos, lavados com água destilada, secos e analisados por meio de difração de raios X. Cabe ressaltar que, devido à formação desses precipitados, a composição final da solução obtida nesse teste, não representa o total de íons removidos da rocha alterada.

\subsection{AM-2 - Ensaio de alteração intermitente}

Para de fato analisar a concentração total dos íons removidos das rochas, tanto a solução de alteração, quanto os precipitados formados no balão de vidro, foram analisados quimicamente por absorção atômica (ASS-3 da Carl Zeiss) e fotometria de chama (modelo B295II da Micronal). No caso das soluções, estas foram filtradas e recolhidas após determinado número de ciclos de lixiviação (Tabela 2). Os precipitados foram, por sua vez, dissolvidos com $\mathrm{HCl}$ concentrado, para posterior análise. Em cada ciclo de lixiviação igual volume de água destilada foi sempre reposto no interior do balão, reiniciando-se o processo. Como o aparato utilizado era um pouco menor que o utilizado em AM-1, colocou-se uma quantidade equivalente menor de rocha carbonática $(200 \mathrm{~g})$ e de água destilada $(650 \mathrm{~mL})$; o tempo gasto em cada etapa de lixiviação também foi em média de $2 \mathrm{~h}$, porém o volume da solução de percolação foi menor (em torno de $150 \mathrm{~mL}$ ). O tempo de funcionamento do soxhlet nesse experimento foi em torno de 281 horas.

Tabela 2 - Número de ciclos de lixiviação das soluções analisadas em AM-2.

\begin{tabular}{cccccccc}
\hline \multirow{2}{*}{$\begin{array}{c}\text { Amostra } \\
n^{\circ} \text { de lixiviações }\end{array}$} & $A M-2 a$ & $A M-2 b$ & $A M-2 c$ & $A M-2 d$ & $A M-2 e$ & $A M-2 f$ & $A M-2 g$ \\
\cline { 2 - 7 } & 2 & 4 & 8 & 16 & 36 & 65 & 150 \\
\hline
\end{tabular}

\subsection{Análise dos dados}

A taxa de mobilidade relativa dos principais íons presentes na rocha carbonática foi analisada por meio do cálculo da razão entre os principais elementos maiores da rocha sã e da solução de lixiviação (HYPOLITO 1972, SIMÃO \& SILVA 1999), segundo a equação (1).

$$
M R=\frac{A_{S}}{A_{R}} * 100 \quad \text { (equação 1) }
$$

onde $A_{S}$ é a concentração do elemento na solução e $A_{R}$, a concentração do mesmo elemento na rocha.

As rochas alteradas no interior do cartucho do experimento AM-1 foram secas a $60^{\circ} \mathrm{C}$ por $24 \mathrm{~h}$ e separadas em três sub-amostras, conforme a posição em que permaneceram no interior do soxhlet
(A, B e C). Estas foram analisadas quimicamente, a partir de métodos gravimétrico para determinações de $\mathrm{SiO}_{2}$ e perda ao fogo, espectrofotométrico para $\mathrm{TiO}_{2}$ e $\mathrm{P}_{2} \mathrm{O}_{5}$ (Micronal, modelo B295II), volumétrico por titulação com dicromato de potássio para $\mathrm{Fe}_{2} \mathrm{O}_{3}$, fotometria de chama (Micronal, modeloB262) para $\mathrm{Na}^{+}$e $\mathrm{K}^{+}$, titulação complexométrica com EDTA para $\mathrm{Ca}^{2+}, \mathrm{Mg}^{2+} \mathrm{e} \mathrm{Al}^{3+}$, e absorção atômica (Carl Zeiss, modelo AAS-3) para os elementos menores e traços $(<1 \%)$.

A partir dos resultados das análises químicas, avaliou-se a evolução do intemperismo artificial das rochas carbonáticas por meio da análise da fração molar, proposta por HYPOLITO (1972). A fração molar $\left(X_{i}\right)$ relaciona o número de mol de um determinado constituinte $\left(n_{i}\right)$ com a soma de uma mistura molar $\left(n_{1}+n_{2}+n_{3}\right)$ dos principais constituintes da rocha analisada (equação 2). 


$$
\begin{aligned}
& \mathrm{X}_{\mathrm{i}}=\frac{\mathrm{n}_{\mathrm{i}}}{n_{1}+n_{2}+n_{3}} \quad \text { (equação 2) } \\
& \text { onde } 0 \leq X_{i} \leq 1 \text { e } \Sigma X_{i}=1
\end{aligned}
$$

Para essa análise foram escolhidos como principais constituintes $\mathrm{CaO}, \mathrm{MgO}$ e $\mathrm{SiO}_{2}$. A partir da determinação das diferenças entre as frações molares de cada constituinte nas rochas sã e alterada, pôde-se avaliar qual deles foi mais removido ou acumulado durante o processo analisado.

\section{RESULTADOS E DISCUSSÃO}

\subsection{Análise das soluções de lixiviação}

A solução final obtida em AM-1 apresentou concentração iônica menor que AM-2 (Tabela 3), devido ao fato dos precipitados formados terem sido separados para a análise química. $\mathrm{O} \mathrm{pH}$ final da solução de AM-1 foi de cerca de 8 , enquanto de AM-2 aumentou conforme o número de lixiviações, de 5 para em torno de 7 .

Uma vez que a solução de lixiviação saturava-se rapidamente formando precipitados (em AM-2 os precipitados formaram-se a partir da etapa $c$, ou seja, depois de 8 ciclos de lixiviação no soxhlet), para quantificar o total de íons lixiviados da rocha, os materiais neoformados em AM-2 foram dissolvidos e analisados juntamente com as soluções finais. Por isso, verificou-se que as concentrações totais dos íons lixiviados das soluções das etapas finais ( $c$ a $g$ ) apresentaram-se bem maiores que àquelas observadas em AM-1.

Tabela 3 - Concentrações de íons $\left(\mathrm{mg}_{\mathrm{L}} \mathrm{L}^{-1}\right)$ e valores de $\mathrm{pH}$ determinados nas soluções de AM-1 e AM-2

\begin{tabular}{|c|c|c|c|c|c|c|c|c|c|}
\hline \multirow{2}{*}{ Lixívia } & \multirow{2}{*}{$A M-1$} & \multicolumn{8}{|c|}{$A M-2$} \\
\hline & & $a$ & $b$ & $c$ & $d$ & $e$ & $f$ & $g$ & Total (\%) \\
\hline ions & $\left(m g \cdot L^{-1}\right)$ & $\left(m g \cdot L^{-1}\right)$ & $\left(m g \cdot L^{-1}\right)$ & $\left(m g \cdot L^{-1}\right)$ & $\left(m g \cdot L^{-1}\right)$ & $\left(m g \cdot L^{-1}\right)$ & $\left(m g \cdot L^{-1}\right)$ & $\left(m g \cdot L^{-1}\right)$ & $\left(m g \cdot L^{-1}\right)$ \\
\hline $\mathrm{Ca}^{2+}$ & $\begin{array}{c}75,29 \\
(0,06 \%)\end{array}$ & $\begin{array}{c}31,15 \\
(0,03 \%)\end{array}$ & $\begin{array}{c}31,15 \\
(0,03 \%)\end{array}$ & $\begin{array}{c}132,31 \\
(0,11 \%)\end{array}$ & $\begin{array}{c}280,38 \\
(0,23 \%)\end{array}$ & $\begin{array}{c}800,39 \\
(0,66 \%)\end{array}$ & $\begin{array}{l}1.117,00 \\
(0,92 \%)\end{array}$ & $\begin{array}{c}2.803,93 \\
(2,31 \%)\end{array}$ & $\begin{array}{l}5.196,31 \\
(4,29 \%)\end{array}$ \\
\hline$M g^{2+}$ & $\begin{array}{c}14,71 \\
(0,24 \%)\end{array}$ & $\begin{array}{c}5,77 \\
(0,10 \%)\end{array}$ & $\begin{array}{c}6,15 \\
(0,11 \%)\end{array}$ & $\begin{array}{c}8,85 \\
(0,15 \%)\end{array}$ & $\begin{array}{c}13,46 \\
(0,23 \%)\end{array}$ & $\begin{array}{c}31,00 \\
(0,53 \%)\end{array}$ & $\begin{array}{c}60,61 \\
(1,04 \%)\end{array}$ & $\begin{array}{c}102,07 \\
(1,74 \%)\end{array}$ & $\begin{array}{c}227,91 \\
(3,90 \%)\end{array}$ \\
\hline $\mathrm{Na}^{+}$ & $\begin{array}{c}29,4 \\
(10,86 \%)\end{array}$ & $\begin{array}{c}0,65 \\
(0,25 \%)\end{array}$ & $\begin{array}{c}0,58 \\
(0,22 \%)\end{array}$ & $\begin{array}{c}1,81 \\
(0,70 \%)\end{array}$ & $\begin{array}{c}6,92 \\
(2,68 \%)\end{array}$ & $\begin{array}{c}18,46 \\
(7,15 \%)\end{array}$ & $\begin{array}{c}10,00 \\
(3,87 \%)\end{array}$ & $\begin{array}{c}26,92 \\
(10.42 \%)\end{array}$ & $\begin{array}{c}65,34 \\
(25,29 \%)\end{array}$ \\
\hline$K^{+}$ & $\begin{array}{c}2,35 \\
(0,32 \%)\end{array}$ & $\begin{array}{c}0,23 \\
(0,03 \%)\end{array}$ & $\begin{array}{c}0,42 \\
(0,06 \%)\end{array}$ & $\begin{array}{c}0,46 \\
(0,07 \%)\end{array}$ & $\begin{array}{c}0,48 \\
(0,07 \%)\end{array}$ & $\begin{array}{c}1,54 \\
(0,22 \%)\end{array}$ & $\begin{array}{c}3,46 \\
(0,50 \%)\end{array}$ & $\begin{array}{c}9,62 \\
(1,39 \%)\end{array}$ & $\begin{array}{c}16,21 \\
(2,34 \%)\end{array}$ \\
\hline $\mathrm{Sr}^{2+}$ & $\begin{array}{c}5,06 \\
(0,86 \%)\end{array}$ & $\begin{array}{c}0,31 \\
(0,06 \%)\end{array}$ & $\begin{array}{c}0,08 \\
(0,01 \%)\end{array}$ & $\begin{array}{c}2,15 \\
(0,38 \%)\end{array}$ & $\begin{array}{c}2,77 \\
(0,50 \%)\end{array}$ & $\begin{array}{c}12,11 \\
(2,17 \%)\end{array}$ & $\begin{array}{c}20,34 \\
(3,63 \%)\end{array}$ & $\begin{array}{c}53,15 \\
(9,50 \%)\end{array}$ & $\begin{array}{c}90,91 \\
(16,26 \%)\end{array}$ \\
\hline$A l^{3+}$ & $\begin{array}{c}0,26 \\
(0,01 \%)\end{array}$ & $\begin{array}{c}1,73 \\
(0,06 \%)\end{array}$ & $\begin{array}{c}1,31 \\
(0,04 \%)\end{array}$ & $\begin{array}{c}0,99 \\
(0,03 \%)\end{array}$ & $\begin{array}{c}3,27 \\
(0,11 \%)\end{array}$ & $\begin{array}{c}7,35 \\
(0,25 \%)\end{array}$ & $\begin{array}{c}7,65 \\
(0,26 \%)\end{array}$ & $\begin{array}{c}14,69 \\
(0,49 \%)\end{array}$ & $\begin{array}{c}36,99 \\
(1,24 \%)\end{array}$ \\
\hline$B a^{2+}$ & $\begin{array}{c}2,2 \\
(0,48 \%) \\
\end{array}$ & $\begin{array}{c}0 \\
(0 \%) \\
\end{array}$ & $\begin{array}{c}0,64 \\
(0,15 \%) \\
\end{array}$ & $\begin{array}{c}0,27 \\
(0,06 \%)\end{array}$ & $\begin{array}{c}1,26 \\
(0,29 \%) \\
\end{array}$ & $\begin{array}{c}4,99 \\
(1,14 \%) \\
\end{array}$ & $\begin{array}{c}24,35 \\
(5,58 \%) \\
\end{array}$ & $\begin{array}{c}21,36 \\
(4,89 \%) \\
\end{array}$ & $\begin{array}{c}52,87 \\
(12,11 \%)\end{array}$ \\
\hline$p H$ & 7,82 & 5,35 & 6,90 & 6,20 & 6,68 & 6,79 & 6,80 & 6,90 & \\
\hline
\end{tabular}
$(a-g)$. Os valores entre parêntesis correspondem à \% em massa dos íons removidos das rochas alteradas.

Em geral, apesar do $\mathrm{Ca}^{2+}, \mathrm{Mg}^{2+} \mathrm{e} \mathrm{Na}^{+}$apresentarem maiores concentrações nas soluções de lixiviação dos dois experimentos, esses íons não foram os mais remobilizados da rocha original. Os dados em \% de massa dos íons removidos das rochas em AM-2 revelaram que, além da remoção iônica aumentar com os ciclos de alteração, os íons mais mobilizados foram $\mathrm{Na}^{+}, \mathrm{Sr}^{2+}, \mathrm{Ba}^{2+}, \mathrm{Ca}^{2+}$ e $\mathrm{Mg}^{2+}$.

Considerando os minerais identificados na rocha sã (calcita, dolomita, muscovita e quartzo), o aumento considerável do $\mathrm{Ca}^{2+}$ nas soluções de altera- ção em todas as etapas indica que a calcita da rocha está continuamente sendo atacada, assim como o $\mathrm{Mg}^{2+}$ da dolomita. Por outro lado, o aumento de $\mathrm{Na}^{+}$ e $\mathrm{K}^{+}$nas soluções de lixiviação indica que a muscovita é a provável fonte desses íons, enquanto o $\mathrm{Sr}^{2+}$ e o $\mathrm{Ba}^{2+}$ podem vir da alteração da própria calcita, uma vez que podem estar presentes nesse mineral como co-precipitados (KOJIMA et al. 1997).

Em AM-2 a cada ciclo de lixiviação houve um aumento crescente e linear de íons lixiviados (principalmente $\mathrm{Ca}^{2+}, \mathrm{Mg}^{2+} \mathrm{e} \mathrm{Sr}^{2+}$ ), indicando que, 
sob as condições impostas pelo experimento, a dissolução da calcita na rocha alterada foi contínua e aumentou de forma linear (Figura 2).
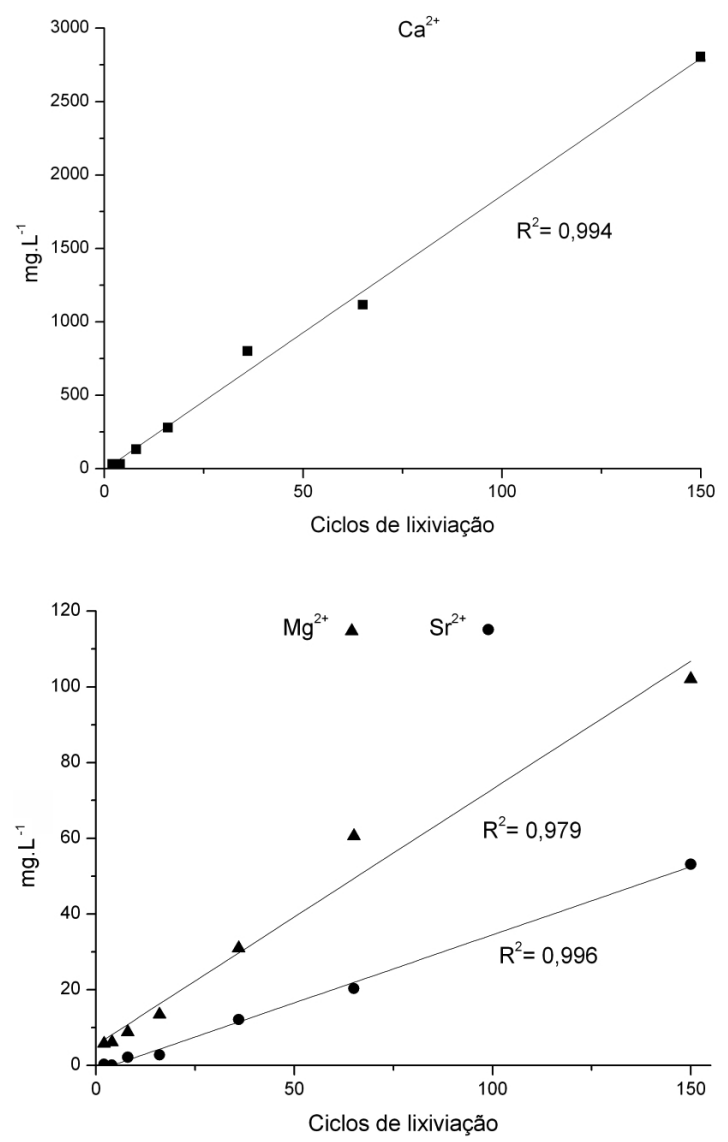

Figura 2 - Teores de $\mathrm{Ca}^{2+}, \mathrm{Mg}^{2+} \mathrm{e} \mathrm{Sr}^{2+}$ determinados nas soluções de AM-2 em função do número de ciclos de lixiviação.

$\mathrm{O}$ fato dessas curvas não atingirem o equilíbrio deve-se ao fato dos dados experimentais incluírem, além da concentração dos íons da solução, a dos minerais neoformados, uma vez que essa fração também corresponde à fase lixiviada da rocha alterada.

Os precipitados em AM-1 e AM-2 começaram a se formar provavelmente quando a solução de lixiviação atingiu a saturação em carbonato, ou seja, após cerca de 8 ciclos de lixiviação.

A análise do difratograma de raios $\mathrm{X}$ dos precipitados formados em AM-1 apresentou picos intensos e nítidos, característicos da calcita. Também foram identificados picos incipientes de prováveis filossilicatos de 10 e $12 \AA$. Ressalta-se que para confirmar a presença desses minerais seria necessário realizar novos ensaios e análises de DRX nos precipitados formados.
Esses picos incipientes podem estar relacionados à presença de paligorsquita (espaçamento basal de 10,3 $\AA$ ) e de sepiolita (espaçamento basal de $12 \AA$ ), uma vez que esses minerais estão comumente associados a calcitas precipitadas em ambiente com pouco $\mathrm{Mg}^{2+}$ (low Mg-calcite) (WATTS 1980). MEUNIER (2005) também afirma que a sepiolita pode se formar juntamente com carbonatos e gipso, precipitando diretamente de soluções durante a evaporação.

PLYAK \& GÜVEN (2000) constataram, por sua vez, que os ambientes alcalino-carbonáticos contendo alumínio e silício são favoráveis à neoformação da paligorsquita autigênica.

Uma vez que a sepiolita está associada à paligorsquita nos depósitos sedimentares (marinho normal, marinho hiper-salino e até lacustre) do mundo inteiro seria provável que ambos fossem produtos de processos de formação similares (ISPHORDING 1973, BLAIR \& CONKO 2011). Conforme esses autores, a formação da paligorsquita assim como da sepiolita, também deve ser sensível às condições de $\mathrm{pH}$ (em torno de 8), concentração de sílica e atividade de $\mathrm{Mg}^{2+}$. Da mesma forma, acredita-se que as soluções de percolação obtidas nesse trabalho ficaram parecidas com as de uma lagoa salina com intensa evaporação; por esse motivo provavelmente houve condições para ocorrer a precipitação da calcita e dos possíveis argilominerais.

Verificou-se presença significativa de $\mathrm{Sr}^{2+} \mathrm{e}$ $\mathrm{Ba}^{2+}$ nas soluções de lixiviação de AM-2, em relação à AM-1. Estima-se que parte desses íons tenha sido removida da solução de AM-1 por precipitação. Por outro lado, essa afirmação sugere que entre os produtos neoformados estivessem presentes minerais do grupo da aragonita, uma vez que esses íons se cristalizam com a estrutura desse mineral. No entanto, o único mineral carbonático identificado no DRX foi a calcita.

Esse comportamento já foi observado por KITANO et al. (1971) nos estágios iniciais de formação de calcitas muito finas. No estágio de nucleação os precipitados de carbonato de cálcio nunca são puros, podendo conter grandes quantidades de carbonato de bário e de estrôncio; conforme os autores, dentre esses dois cátions, $\mathrm{o} \mathrm{Ba}^{2+}$ possui maior influência na precipitação de $\mathrm{CaCO}_{3}$ que o $\mathrm{Sr}^{2+}$.

A precipitação da calcita ou aragonita, por sua vez, é fortemente influenciada pela presença de $\mathrm{Mg}^{2+}$ na solução (KITANO \& HOOD 1962, MULLER et al. 1972, DAVIS et al. 2000, CHOUDENS-SÁNCHEZ \& GONZÁLEZ 2009). A incorporação de $\mathrm{Mg}^{2+}$ na estrutura cristalina da calcita aumenta proporcionalmente com a concentração 
de $\mathrm{Mg}^{2+}$ na solução, inibindo a taxa de crescimento desse mineral (DAVIS et al. 2000). Todavia, a presença de $\mathrm{Mg}^{2+}$ em solução não afeta a taxa de crescimento da aragonita, uma vez que esse íon não pode ser incorporado na estrutura desse mineral (JIMÉNEZ-LÓPEZ et al. 2004).

Assim, sob baixa razão de $\mathrm{Mg} / \mathrm{Ca}$ na solução, a calcita é a principal fase mineral a se precipitar; com o aumento dessa razão a formação da aragonita passa a predominar, devido à inibição da formação da calcita (CHOUDENS-SÁNCHEZ \&
GONZÁLEZ 2009). De acordo com MULLER et al. (1972), quando a razão $\mathrm{Mg} / \mathrm{Ca}$ é muito elevada podem-se formar carbonatos magnesianos hidratados (hidromagnesita, nesquehonita).

Nas soluções de lixiviação obtidas em AM-1 e nas etapas iniciais de AM-2 ( $a$ e $b$ ), observou- se que a razão de $\mathrm{Mg} / \mathrm{Ca}$ permaneceu baixa (em torno de 0,3 ). A partir da etapa $c$, quando se iniciou a precipitação de carbonatos, verificou-se que essa razão começa a diminuir, devido à entrada cada vez maior de $\mathrm{Ca}^{2+}$ na solução de lixiviação (Ta-

Tabela 4 - Valores das razões molares de $\mathrm{Mg} / \mathrm{Ca}$ das soluções de alteração experimental (AM-1 e AM-2 a-g).

\begin{tabular}{c|c|cccccccc}
\hline & \multirow{2}{*}{} & AM-1 & \multicolumn{7}{|c}{ AM-2 } \\
\cline { 3 - 9 } & & $\mathrm{a}$ & $\mathrm{b}$ & $\mathrm{c}$ & $\mathrm{d}$ & $\mathrm{e}$ & $\mathrm{f}$ & $\mathrm{g}$ \\
\hline $\mathrm{Mg} / \mathrm{Ca}$ & 0,326 & 0,308 & 0,329 & 0,111 & 0,08 & 0,065 & 0,090 & 0,061 \\
\hline
\end{tabular}

Tabela 5 - Concentrações (mg. $\mathrm{L}^{-1}$ ) e valores de mobilidade relativa (MR) dos principais constituintes analisados na rocha sã e nas soluções de alteração dos experimentos AM-1 e AM-2 $a-g$.

\begin{tabular}{|c|c|c|c|c|c|c|c|}
\hline Amostra & $\mathrm{Ca}$ & $M g$ & $A l$ & $\mathrm{Na}$ & $K$ & $S r$ & $B a$ \\
\hline Rocha (\%) & 34,900 & 1,682 & 0,857 & 0,074 & 0,199 & 0,161 & 0,130 \\
\hline \multicolumn{8}{|c|}{ Solução de AM-1 } \\
\hline$A M-1\left(m g \cdot L^{-1}\right)$ & 75,29 & 14,71 & 0,26 & 29,40 & 2,35 & 5,06 & 2,20 \\
\hline$M R$ & 0,022 & 0,087 & 0,003 & 3,962 & 0,118 & 0,315 & 0,176 \\
\hline \multicolumn{8}{|c|}{$\mathrm{Na}>\mathrm{Sr}>\mathrm{Ba}>\mathrm{K}>\mathrm{Mg}>\mathrm{Ca}>\mathrm{Al}$} \\
\hline \multicolumn{8}{|c|}{ Soluções de AM-2 } \\
\hline$a\left(m g \cdot L^{-1}\right)$ & 31,15 & 5,77 & 1,73 & 0,65 & 0,23 & 0,31 & 0,00 \\
\hline$M R$ & 0,009 & 0,034 & 0,020 & 0,088 & 0,012 & 0,019 & 0,00 \\
\hline \multicolumn{8}{|c|}{$\mathrm{Na}>\mathrm{Mg}>\mathrm{Al}>\mathrm{Sr}>\mathrm{K}=\mathrm{Ca}$} \\
\hline$b\left(m g \cdot L^{-1}\right)$ & 31,15 & 6,15 & 1,31 & 0,58 & 0,42 & 0,08 & 0,64 \\
\hline$M R$ & 0,009 & 0,037 & 0,015 & 0,078 & 0,021 & 0,005 & 0,051 \\
\hline \multicolumn{8}{|c|}{$\mathrm{Na}>\mathrm{Ba}>\mathrm{Mg}>\mathrm{K}>\mathrm{Al}>\mathrm{Ca}>\mathrm{Sr}$} \\
\hline$c\left(m g \cdot L^{-1}\right)$ & 132,31 & 8,85 & 0,99 & 1,81 & 0,46 & 2,15 & 0,27 \\
\hline$M R$ & 0,038 & 0,053 & 0,012 & 0,244 & 0,023 & 0,134 & 0,022 \\
\hline \multicolumn{8}{|c|}{$\mathrm{Na}>\mathrm{Sr}>\mathrm{Mg}>\mathrm{Ca}>\mathrm{K}>\mathrm{Ba}>\mathrm{Al}$} \\
\hline$d\left(m g \cdot L^{-1}\right)$ & 280,38 & 13,46 & 3,27 & 6,92 & 0,48 & 2,77 & 1,26 \\
\hline$M R$ & 0,080 & 0,080 & 0,038 & 0,933 & 0,024 & 0,172 & 0,101 \\
\hline \multicolumn{8}{|c|}{$\mathrm{Na}>\mathrm{Sr}>\mathrm{Ba}>\mathrm{Mg}=\mathrm{Ca}>\mathrm{Al}>\mathrm{K}$} \\
\hline$e\left(m g \cdot L^{-1}\right)$ & 800,39 & 31,00 & 7,35 & 18,46 & 1,54 & 12,11 & 4,99 \\
\hline$M R$ & 0,229 & 0,184 & 0,086 & 2,488 & 0,077 & 0,754 & 0,399 \\
\hline \multicolumn{8}{|c|}{$\mathrm{Na}>\mathrm{Sr}>\mathrm{Ba}>\mathrm{Ca}>\mathrm{Mg}>\mathrm{Al}>\mathrm{K}$} \\
\hline$f\left(m g \cdot L^{-1}\right)$ & 1117,00 & 60,61 & 7,65 & 10,00 & 3,46 & 20,34 & 24,35 \\
\hline$M R$ & 0,320 & 0,360 & 0,089 & 1,348 & 0,174 & 1,266 & 1,948 \\
\hline \multicolumn{8}{|c|}{$\mathrm{Ba}>\mathrm{Na}>\mathrm{Sr}>\mathrm{Mg}>\mathrm{Ca}>\mathrm{K}>\mathrm{Al}$} \\
\hline$g\left(m g \cdot L^{-1}\right)$ & 2803,93 & 102,07 & 14,69 & 26,92 & 9,62 & 53,15 & 21,36 \\
\hline$M R$ & 0,803 & 0,607 & 0,171 & 3,628 & 0,483 & 3,308 & 1,709 \\
\hline \multicolumn{8}{|c|}{$\mathrm{Na}>\mathrm{Sr}>\mathrm{Ba}>\mathrm{Ca}>\mathrm{Mg}>\mathrm{K}>\mathrm{Al}$} \\
\hline
\end{tabular}


bela 4), favorecendo ainda mais a precipitação de calcita.

Como visto, apesar da maior quantidade de $\mathrm{Ca}^{2+}$ em solução, este não foi o elemento mais mobilizado, uma vez que seu teor na rocha era muito mais elevado em relação aos outros constituintes. Corroborando os dados de porcentagem em massa (Tabela 3), os valores de mobilidade relativa (Tabela 5) determinados em todas as soluções analisadas revelaram que o íon com maior mobilidade relativa foi o sódio.

Os dados obtidos na solução de AM-1 revelaram que os elementos mais removidos foram: $\mathrm{Na}^{+}>\mathrm{Sr}^{2+}>\mathrm{Ba}^{2+}>\mathrm{K}^{+}>\mathrm{Mg}^{2+}>\mathrm{Ca}^{2+}>\mathrm{Al}^{3+}$. Cabe ressaltar novamente que nessa interpretação não se considerou os precipitados, que se formaram a partir de uma determinada concentração, saindo da solução de lixiviação.

A mobilidade relativa dos íons analisados em AM-2 no início dos ciclos de lixiviação $(a$ e $b)$ seguiu a mesma ordem observada em AM-1. No entanto, a partir da etapa $c$, com a formação de precipitados, os valores de MR de $\mathrm{Ca}^{2+}$ aumentaram, posicionando esse íon entre os mais móveis, junto com $\mathrm{Na}^{+}, \mathrm{Sr}^{2+}$ e $\mathrm{Ba}^{2+}$.

De forma geral, a mobilidade relativa (MR) de todos os constituintes analisados nas soluções de AM-2 aumentou de acordo com os números de lixiviações (Figura 3).

\subsection{Análise das rochas alteradas}

A análise por difração de raios $\mathrm{X}$ das rochas alteradas em AM-1 revelou a presença de um mineral secundário, caulinita, além daqueles que já faziam parte da composição da rocha original (calcita, dolomita, quartzo e muscovita). Verificou-se também maior grau de alteração nas rochas que permaneceram no nível intermediário (parte B do cartucho), submetidas à variação constante do nível d'água. As amostras que ficaram na parte aérea (A) parecem ter sido medianamente alteradas e as que permaneceram submersas durante todo o experimento (C) foram as que menos sofreram alteração. Em A e B, as rochas ficaram mais pulverulentas e esbranquiçadas, enquanto em $\mathrm{C}$, permaneceram mais rígidas e com coloração semelhante à rocha sã (cinza escuro).

Os resultados das análises químicas (Tabela 6) também indicaram que as rochas do nível B foram quimicamente mais alteradas, por conta da remoção pronunciada de cálcio e aumento relativo nos teores de magnésio e silício.

Para verificar se houve aumento $(+)$ ou diminuição (-) desses componentes nas rochas analisadas, as frações molares $(X)$ das amostras alteradas (Tabela 7) foram comparadas com as da rocha sã (Tabela 8).

Dentre os constituintes analisados verificou-se de modo geral que o $\mathrm{CaO}$ foi realmente o componente mais remobilizado das rochas, enquanto $\mathrm{SiO}_{2}$ e $\mathrm{MgO}$ sofreram um acúmulo relativo.

Tabela 6 - Principais óxidos analisados nas rochas sã e alteradas (A, B e C) em AM-1.

\begin{tabular}{ccccc}
\hline Óxidos & Rocha sã & A (zona aerada) & B (zona intermediária) & C (zona saturada) \\
& & & $\%$ & \\
\hline $\mathrm{CaO}$ & 48,88 & 40,68 & 36,49 & 39,76 \\
$\mathrm{MgO}$ & 2,79 & 6,05 & 8,04 & 6,56 \\
$\mathrm{SiO}_{2}$ & 4,64 & 11,66 & 14,02 & 10,84 \\
$\mathrm{Fe}_{2} \mathrm{O}_{3}$ & n.d. & 0,91 & 1,07 & 0,90 \\
$\mathrm{TiO}_{2}$ & 0,05 & 0,11 & 0,15 & 0,15 \\
$\mathrm{~K}_{2} \mathrm{O}$ & 0,24 & 0,47 & 0,48 & 0,42 \\
$\mathrm{Na}_{2} \mathrm{O}$ & 0,10 & 0,13 & 0,13 & 0,30 \\
$\mathrm{SrO}$ & 0,19 & 0,53 & 0,48 & 0,53 \\
$\mathrm{PF}$ & 40,59 & 36,50 & 37,46 & 36,75 \\
\hline $\mathrm{Total}$ & 97,48 & 97,04 & 98,32 & 96,21 \\
\hline
\end{tabular}



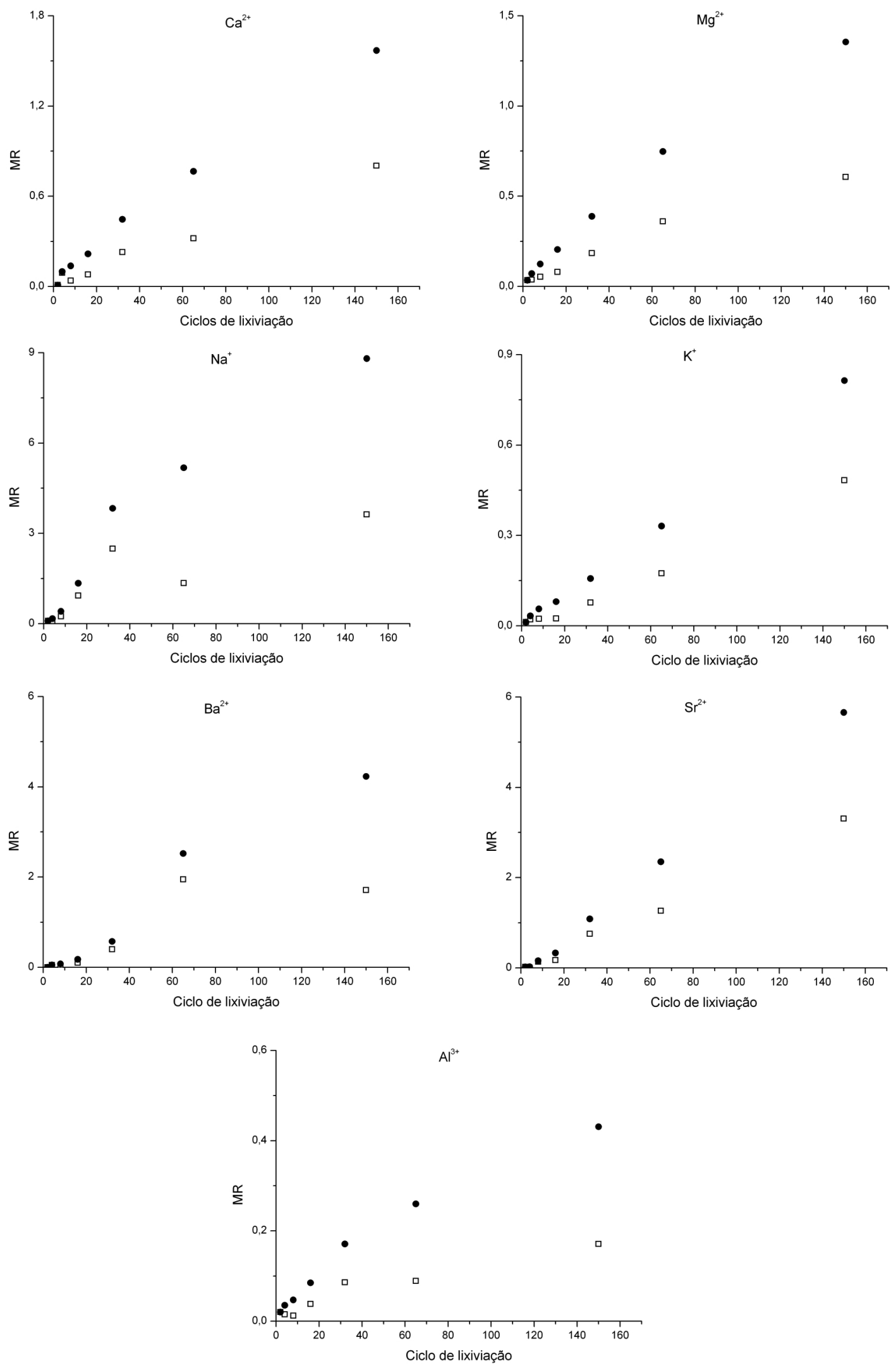

Figura 3 - Curvas dos valores de MR absolutos ( $\square$ ) e cumulativa $(\bullet)$ dos íons analisados nas soluções de AM-2 (a-g) 
Tabela 7 - Número de mol (n) dos componentes estudados e suas respectivas frações molares (X) dada em \%.

\begin{tabular}{ccccc}
\hline & Rocha sã & A (zona aerada) & B (zona intermediária) & C (zona saturada) \\
\hline \multicolumn{5}{c}{ Número de mol (n) } \\
\hline $\mathrm{CaO}$ & 1,55 & 1,24 & 1,11 & 1,24 \\
$\mathrm{MgO}$ & 0,12 & 0,26 & 0,34 & 0,28 \\
$\mathrm{SiO}$ & 0,14 & 0,33 & 0,40 & 0,32 \\
\hline \multicolumn{5}{c}{ Fração molar $(X \%)$} \\
$\mathrm{CaO}$ & 85,62 & 67,83 & 60,02 & 67,35 \\
$\mathrm{MgO}$ & 6,79 & 14,04 & 18,42 & 15,48 \\
$\mathrm{SiO}$ & 7,59 & 18,13 & 21,56 & 17,16 \\
\hline
\end{tabular}

Tabela 8 - Variação das frações molares entre as rochas alteradas e sã. O sinal (+) indica que houve aumento da fração molar e (-), diminuição.

\begin{tabular}{cccc}
\hline & A (zona aerada) & B (zona intermediária) & $C$ (zona saturada) \\
\hline $\mathrm{CaO}$ & $-17,79$ & $-25,60$ & $-18,27$ \\
$\mathrm{MgO}$ & $+7,25$ & $+11,63$ & $+8,69$ \\
$\mathrm{SiO}$ & $+10,54$ & $+13,97$ & $+9,57$ \\
\hline
\end{tabular}

\section{CONSIDERAÇÕES FINAIS}

O uso do aparato soxhlet no estudo da alteração experimental das rochas carbonáticas da $\mathrm{Ca}$ verna das Pérolas permitiu verificar que:

1. as soluções de lixiviação apresentaram em geral elevadas concentrações de $\mathrm{Ca}^{2+}$ e $\mathrm{Mg}^{2+}$, no entanto estes não foram os íons mais removidos da rocha;

2. os íons mais livixiados da rocha carbonática durante o processo de alteração experimental foram $\mathrm{Na}^{+}, \mathrm{Sr}^{2+}$ e $\mathrm{Ba}^{2+}$;

3. o experimento que simulou a alteração contínua (AM-1) indicou a seguinte ordem de mobilidade relativa de íons: $\mathrm{Na}>\mathrm{Sr}>$ $\mathrm{Ba}>\mathrm{K}>\mathrm{Mg}>\mathrm{Ca}>\mathrm{Al}$;

4. ao considerar-se os íons precipitados (experimento de alteração intermitente - AM-2), obteve-se a seguinte ordem de mobilidade relativa dos íons: $\mathrm{Na}>\mathrm{Sr}>\mathrm{Ba}>\mathrm{Ca}>\mathrm{Mg}$ $>K>A l$;

5. apesar das concentrações de $\mathrm{Sr}^{2+}$ e $\mathrm{Ba}^{2+}$ aumentarem com os ciclos de lixiviação das rochas, não houve precipitação de minerais do grupo da aragonita; o fator determinante foi a razão de $\mathrm{Mg} / \mathrm{Ca}$, que permaneceu sempre baixa, favorecendo a precipitação de calcita e a co-precipitação de $\mathrm{Ba}^{2+}$ e $\mathrm{Sr}^{2+}$, retirando-os da solução de lixiviação;
6. as amostras de rochas submetidas a constantes mudanças de nível das soluções de percolação foram as que mais sofreram alteração química;

7. com o aumento do processo de lixiviação, formou-se nas rochas alteradas minerais secundários como a caulinita.

\section{REFERÊNCIAS}

BARBIERI, A.J. 1993. Depósitos minerais secundários das cavernas Santana, Pérolas e Lage Branca, Município de Iporanga - São Paulo. Instituto de Geociências da Universidade de São Paulo, Dissertação de Mestrado, 96p.

BLAIR, F.J.; CONKO, K. 2011. Environmental influences on the occurrences of sepiolite and palygorskite: a brief review. In: E. GALAN, E.; A. SINGER (eds). Developments in palygorskite-sepriolite research - a new Outlook of these nanomaterials. Amsterdam, Elsevier, 3: 69-83.

BUHMANN, D.; DREYBRODT, W. 1985. The kinetics of calcite dissolution and precipitation in geologically relevant situations of karst areas - 1. Open system. Chemical Geology, 48: 189-211. 
CAMPANHA, G.A.C. 1991. Tectônica proterozóica no alto e médio Vale do Ribeira, estados de São Paulo e Paraná. Instituto de Geociências, Universidade de São Paulo, Tese de Doutoramento, $295 \mathrm{p}$.

CHOUDENS-SÁNCHEZ, V.; GONZÁLEZ, L.A. 2009. Calcite and aragonite precipitation under controlled instantaneous supersaturation: elucidating the role of $\mathrm{CaCO}_{3}$ saturation state anda $\mathrm{Mg} / \mathrm{Ca}$ ratio on calcium carbonate polymorphism. Journal of Sedimentay Research, 79: 363-376.

COSTA, A.P.L.; NOGUEIRA NETO, J.A.; GALEMBECK, T.M.B.; SILVA, Z.C.G.; SIMÃO, J.A. 2010. Rochas ornamentais: alterabilidade dos gnaisses enderbíticos no ensaio de lixiviação contínua através do extrator soxhlet. Holos, 3: 3-12.

CUADROS, J.; CABALLERO, E.; HUERTAS, F.J.; CISNEROS, C.J.; HUERTAS, F.; LINARES, J. 1999. Experimental alteration of volcanic tuff: smectite formation and effect on ${ }^{18} \mathrm{O}$ isotope composition. Clays and Clay Minerals, 47(6): 769-776.

DAVIS, K.J.; DOVE, P.M.; DE YOREO, J.J. 2000. The role of $\mathrm{Mg}^{2+}$ as an impurity in calcite growth. Science, 290: 1134-1137.

DREYBRODT, W.; BUHMANN, D. 1991. A mass transfer model for dissolution and precipitation of calcite from solutions in turbulent motion. Chemical Geology, 90:107-122.

DREYBRODT, W.; LAUCKNER, J.; ZAIHUA, L.; SVENSSON, U.; BUHMANN, D. 1996. The kineyics of the reaction $\mathrm{CO}_{2}+\mathrm{H}_{2} \mathrm{O} \rightarrow \mathrm{H}^{+}$ $+\mathrm{HCO}_{3}{ }^{-}$as one of the rate limiting steps for the dissolution of calcite in the system $\mathrm{H}_{2} \mathrm{O}-$ $\mathrm{CO}_{2}-\mathrm{CaCO}_{3}$. Geochimica et Cosmochimica Acta, 18: 3375-3381.

FORD, D.; WILLIAMS, P. 2007. Karst Hydrology and geomorphology. New Zealand, John Wiley \& sons Ltda., 562p.

FRASCÁ, M.H.B.O.; YAMAMOTO, J.K. 2006. Ageing tests for dimension Stone-experimental studies of granitic rocks from Brazil. In: 10th IAEG International Congress, Nottingham, United Kingdom, 6-10 September, 2006. Paper number 224 .

GOMES, R.L. 2001. Características tecnológicas e alterabilidade dos compartimentos enta- blamento e colunata de derrames basálticos da porção setentrional da Bacia do Paraná. Escola de Engenharia de São Carlos, Universidade de São Paulo, São Carlos, Tese de Doutoramento, 295p.

HOREAU, J.L.; NICOLINI, E.; FRITZ, B.; DELCHER, E. 2005. Signatures géochimiques dês eaux souterraines em milieu basaltique tropical (Ilê de La Réunion). Approche expérimentale. Bulletin de la Société Géologique de France, 176(3): 257-267.

HYPOLITO, R. 1972. Estudo experimental de alteração intempérica de diabásios. Instituto de Geociências, Universidade de São Paulo, Tese de Doutoramento, 103p.

HYPOLITO, R. 1974. Ensaios de alteração intempérica utilizando um novo aparelho: o intemperizador. Boletim do Instituto de Geociências, USP, 5: 65-84.

IPT (Instituto de Pesquisas Tecnológicas do Estado de São Paulo). 1984. Geologia das Folhas Iporanga e Gruta do Diabo. São Paulo, IPT/SICCT/ Pró-Minério. Relatório 22352, VI. 207 p.

ISPHORDING, W. 1973. Discussion of the occurrence and origin of sedimentary palygosrkite-sepiolite deposits. Clays and Clay Minerals, 21: 391-401.

JIMÉNEZ-LÓPEZ,C.; OHMOTO,H.; CABALLERO, E.; ROMANEK, C.S.; HUERTAS, F.J. 2004. Oxygen isotope fractionation in synthetic magnesian calcite. Geochimica et Cosmochimica Acta, 68: 3367-3377.

KARMANN, I.; SANCHEZ, L.E. 1979. Distribuição das rochas carbonáticas e províncias espeleológicas do Brasil. Espeleo-Tema, 13: 105-167.

KITANO, Y.; HOOD, D.W. 1962. Calcium carbonate crystal forms formed from sea water by inorganic process. The Journal of the Oceanographical Society of Japan, 18(3): 141-145.

KITANO, Y.; KANAMORI, N.; OOMORI, T. 1971. Measurements of distribution coefficients of strontium and barium between carbonate precipitate and solution - Abnormally high values of distribution coefficients measured at early stages of carbonate formation. Geochemical Journal, 4: 183-206. 
KOJIMA, T.; NAGAMINE, A.; UENO, N.; UEMIYA, S. 1997. Absortion and fixation of carbon dioxide by rock weathering. Energy Conversion and Management, 38:461-466.

KRYZA， R.; PRELL, M.; CZECHOWSKI, F.; DOMARADZKA, M. 2009. Acidic weathering of carbonate building stones: experimental assessment (preliminary results). Studia Universitatis Babeç-Bolyai, Geologia, 54(1): 33-36.

LEE，M.R.; SMITH， C.L.; GORDON， S.H.; HODSON, M.E. 2006. Laboratory simulation of terrestrial meteorite weathering using the Bensour (LL6) ordinary chondrite. Meteoritics \& Planetary Science, 41(8): 1123-1138.

MARTINEZ, M.I.; WHITE, W.B. 1999. A laboratory investigation of the relative dissolution rates of the Lirio Limestone and the Isla de Mona Dolomite and implications for cave and karst development on Isla de Mona. Journal of Cave and Karst Studies, 61(1): 7-12.

MEUNIER, A. 2005. Clays. New York, Springer Verlag, $1^{a}$ ed. $485 p$.

MULLER, G.; IRION, G.; FORSTNER, V. 1972. Formation and diagenesis of inorganic $\mathrm{Ca}$ $\mathrm{Mg}$ carbonates in the lacustrine environmet. Naturwissenschaften, 4: 158-164.

PEDRO, G. 1964. Contribution a l'étude experimentale de l'alteration géochimique dês roches cristalines. Paris, Institut National de La Recherche Agronomique, 345p.
PLYAK, V.J.; GÜVEN, N. 2000. Clays in caves of the Guadalupe Mountains, New Mexico. Journal of Cave and Karst Studies, 62(2): 120-126.

PRIKRYL, R.; LOKAJICEK, T.; SVOBODOVÁ, J.; WEISHAUPTOVÁ, Z. 2003. Experimental weathering of marlstone form Prední Kopanina (Czech Recpublic) - historical building Stone of Prague. Building and Environment, 38: 1163-1171.

SIMÃO, J.; SILVA, Z.C.G. 1999. Alteration and alterability of the anorthosite from Angola. Ciências da Terra (UNL), 13: 9-22.

WATTS, N.L. 1980. Quaternary pedogenic calcretes from the Kalahari (South Africa): mineralogy, genesis and diagenesis. Sedimentology, 27(6): 661-686.

WHITE, A.F.; BRANTLEY, S. 2003. The effect of time on the weathering of silicate minerals: why do weathering rates differ in the laboratory and field? Chemical Geology, 202: 479-506.

WHITE, A.F.; BULLEN， T.D.; VIVIT, D.V.; SCHULZ, M.S.; CLOW, D.W. 1999. The role of disseminated calcite in the chemical weathering of granitoid rocks. Geochimica et Cosmochimica Acta, 63(13/14): 1939-1953.

WU, J.C.S.; SHEEN, J.D.; CHEN, S.Y.; FAN, Y.C. 2001. Feasibility of CO2 fixation via artificial rock weathering. Industrial \& Engineering Chemistry Research, 40: 3902-3905.

Endereço dos autores:

Mirian Chieko Shinzato - Universidade Federal de São Paulo (UNIFESP), Campus Diadema, Rua Artur Riedel, 275. CEP: 09972-270, Diadema, SP. E-mail: mirianshinzato@hotmail.com

Raphael Hypolito, Alex José Barbieri e Flávio Machado de Souza Carvalho - Instituto de Geociências, Universidade de São Paulo, Rua do Lago, 562. CEP: 05508-080, Cidade Universitária, São Paulo, SP. E-mails: rhypo@igc.usp.br; flavioms@usp.br 